\title{
Dark Energy and Cosmic Speed-Up from Consistent Modified Gravity
}

\author{
Shin'ichi Nojiri \\ Department of Applied Physics, National Defence Academy \\ Hashirimizu Yokosuka 239-8686, Japan \\ email: nojiri@nda.ac.jp, snojiri@yukawa.kyoto-u.ac.jp
}

\section{Sergei D.Odintsov ${ }^{* \dagger}$}

Instituciò Catalana de Recerca i Estudis Avançats (ICREA) and Institut d'Estudis Espacials de Catalunya (IEEC), Edifici Nexus, Gran Capità 2-4, 08034 Barcelona, Spain

email: odintsov@ieec.uab.es

\begin{abstract}
We review some modified gravity models which describe the gravitational dark energy and the possibility of cosmic speed-up. The new consistent version of such theory which contains inverse and HD curvature terms as well as new type of coupling with matter is proposed. The accelerating cosmologies are discussed there. The structure of finite-time (sudden) singularities is investigated.
\end{abstract}

Fourth International Winter Conference on Mathematical Methods in Physics 09 - 13 August 2004

Centro Brasileiro de Pesquisas Fisicas (CBPF/MCT), Rio de Janeiro, Brazil

\author{
* Speaker. \\ ${ }^{\dagger}$ Also at TSPU, Tomsk, Russia
}


The very interesting approach to resolution of dark energy problem which is the challenge for XXI century is related with possible modifications of gravity at large distances. In particular, one can consider gravity with the terms which are inverse on Riemannian invariants (Riemann and Ricci tensor, curvature). The simplest example of that sort is $1 / R$ theory [1] where the growth of (gravitational) dark energy is explained by the expansion. The inverse curvature terms may have Mtheory or higher dimensional origin[2,3]. Even, $1 / R$ model may contain some instabilities or may not pass the solar system tests [4], it exists its consistent modification [5, 6, 7] by higher derivative or logarithmic terms. Moreover, its Palatini version (for recent discussion, see [8]) may probably have less problems. Various (cosmological) aspects of modified gravity with inverse curvature terms have been studied in refs.[9, 10,11, 12]. In the present report we review the modified gravity aspects which are related with dark energy and cosmic speed-up. The new modified gravity model is suggested where some cosmological solutions are given and its future singularity behaviour is discussed.

As an interesting example the action of (large distances) modified gravity may be taken in the following form

$$
S=\frac{1}{\kappa^{2}} \int d^{4} x \sqrt{-g}\left(R-\gamma R^{-n}+L_{m}\right)
$$

Here $\gamma$ is (an extremely small) coupling constant and $n$ is some number. One can work in the original Jordan frame or, using conformal transformation to scalar-tensor gravity, in Einstein frame (see[13]). Without matter and when the curvature is small, the solution of equations of motion may be found [1]. The FRW universe metric in Jordan frame is $d s^{2}=-d t^{2}+a^{2}(t) \sum_{i=1}^{3}\left(d x^{i}\right)^{2}$. The explicit FRW scale factor is

$$
a \sim t^{\frac{(n+1)(2 n+1)}{n+2}}, \quad w=-\frac{6 n^{2}+7 n-1}{3(n+1)(2 n+1)} .
$$

where $w$ is the effective equation of state parameter. If $n>\frac{-1+\sqrt{3}}{2}$ or $-1<n<-\frac{1}{2}$, we find $w<-\frac{1}{3}$ and $\frac{d^{2} a}{d t^{2}}>0$, that is, the universe is expanding with acceleration. The fact that gravity with inverse curvature may provide the gravitational dark energy model responsible for cosmic speed-up attracts the interest to such theory.

If $w<-1$, the universe is shrinking in the expression of $a$ (2). If we replace the direction of time by changing $t$ by $-t$, the universe is expanding but $t$ should be considered to be negative so that the scale factor $a$ should be real. Then there appears a singularity at $t=0$, where the scale factor $a$ diverges as $a \sim(-t)^{\frac{2}{3(w+1)}}$. One may shift the origin of the time by further changing $-t$ with $t_{s}-t$. Hence, in the present universe, $t$ should be less than $t_{s}$ and there will appear the singularity at $t=t_{s}: a \sim\left(t_{s}-t\right)^{\frac{2}{3(w+1)}}$. The future finite-time singularity is of sudden or Big Rip type. Since $w+1=\frac{2(n+2)}{3(n+1)(2 n+1)}$, it follows $w<-1$ when $-1<n<-\frac{1}{2}$. Here it is assumed $n>-1$, so that the Einstein term dominates when the curvature is small.

Let the ideal fluid is taken as the matter with the constant $w: p=w \rho$. From the energy conservation law $0=d \rho / d t+3 H(\rho+p)$, it follows $\rho=\rho_{0} a^{-3(1+w)}$.

When the curvature is small, if $n>-1, f(R)$ may behave as $f(R) \sim-\gamma R^{-n}$. In such a limit, 
an exact solution of the equation of motion is found to be [11]

$$
a=a_{0} t^{h_{0}}, \quad h_{0} \equiv-\frac{2 n}{3(1+w)}, \quad a_{0} \equiv\left[-\frac{-6 \gamma h_{0}}{\rho_{0}} \frac{\left\{(1+2 n)(1+n)-(2+n) h_{0}\right\}}{\left(-6 h_{0}+12 h_{0}^{2}\right)^{n+1}}\right]^{-\frac{1}{3(1+w)}} .
$$

When $n=-1$, the result $h_{0}=\frac{2}{3(1+w)}$ in the Einstein gravity is reproduced. The effective $w_{\text {eff }}$ may be defined by $h_{0}=\frac{2}{3\left(1+w_{\text {eff }}\right)}$. By using (3), one finds $w_{\text {eff }}=-1+\frac{1+w}{n}$. Hence, if $w$ is greater than -1 (effective quintessence or even usual ideal fluid with positive $w$ ), when $n$ is positive, we obtain the effective phantom phase where $w_{\text {eff }}$ is less than -1 . This is different from the case of pure modified gravity (2). When $w_{\text {eff }}<-1$, the curvature tends to become large. In the usual phantom model, the increase of the curvature generates the Big Rip type singularity (for recent discussion, see[14]). In the model (1), however, when the curvature is large, the first Einstein-Hilbert term dominates. Hence, phantom era is transient.

The equation of motion may be written as:

$$
\frac{1}{\kappa^{2}}\left(R_{\mu v}-\frac{1}{2} g_{\mu v}\right)=T_{\mu v}^{\mathrm{dark}}+T_{\mu v}
$$

Here $T_{\mu \nu}^{\text {dark }} \equiv \frac{\gamma}{\kappa^{2}}\left\{-n R_{\mu v} R^{-n-1}-\frac{1}{2} g_{\mu \nu} R^{-n}+n\left(\nabla_{\mu} \nabla_{v}-g_{\mu \nu} \nabla^{2}\right) R^{-n-1}\right\}$. One may regard $T_{\mu \nu}^{\text {dark }}$ as the gravitational dark energy. Due to the identity $\nabla^{\mu}\left(R_{\mu v}-\frac{1}{2} g_{\mu v}\right)=0$, the total energy momentum tensor $T_{\mu \nu}^{\text {dark }}+T_{\mu \nu}$ is conserved. If it is also assumed the conservation of the energy, the dark energy part $T_{\mu \nu}^{\text {dark }}$ is also conserved. Then it looks that there is no flow of the energy between the matter sector and the dark energy one. However, it occurs via the gravity coupling. In the solution (3), since the first Einstein-Hilbert term in (1) is neglected, we find $T_{\mu \nu}^{\text {dark }}=-T_{\mu \nu}$, especialy, $\rho^{\text {dark }}=-\rho$, that is, the variation of the matter energy density $\rho$ is absorbed into the dark energy density $\rho^{\text {dark }}$. When $n<0$ and $w>0$, which gives $w_{\text {eff }}<-1$ in (3) for small curvature, it follows

$$
\rho \sim-\rho^{\text {dark }} \sim \rho_{0}\left(t_{s}-t\right)^{2 n}, \quad \rho_{0} \equiv 6 h_{0} \gamma\left(-6 h_{0}+12 h_{0}^{2}\right)^{-n-1}\left((2 n+1)(n+1)-(n+2) h_{0}\right) .
$$

Comparing it with the case of the Einstein gravity, where $\rho$ behaves as $\rho \sim t^{-2}, \rho$ (5) decreases more rapidly and tends to vanish at $t=t_{s}$. The decrease of $\rho$ generates the increase of $\rho^{\text {dark }}$. Since the first Einstein-Hilbert term (1) is neglected in the solution (3), we may include the Einstein-Hilbert term perturbatively. Using (4), one gets

$$
\rho^{\mathrm{dark}}+\rho=\frac{6 h_{0}^{2}}{\kappa^{2}\left(t_{s}-t\right)^{2}}=\frac{8 n^{2}}{3(1+w)^{2}\left(t_{s}-t\right)^{2}},
$$

which becomes large when $t$ goes to $t_{s}$. Since the usual energy density decreases when the size of the universe increases, Eq.(6) shows that the dark energy $\rho^{\text {dark }}$ becomes large rapidly. Eq.(6) also indicates that one cannot neglect the Einstein term when $t \sim t_{s}$. When the curvature becomes large and the Einstein term dominates, the spacetime behaves as $a \sim t^{\frac{2}{3(w+1)}}$. If we perturbatively include the second $R^{-n}$ term in (1), by using (4)

$$
\rho^{\mathrm{dark}} \sim 6 h_{0}\left((1+n)(1+2 n)-(2+n) h_{0}\right)\left(-6 h_{0}+12 h_{0}^{2}\right)^{-n-1} t^{2 n},
$$

which increases with time (transient period). 
Let us now consider the following consistent modified gravity model composed of two theories[5, 15]:

$$
S=\int d^{4} x \sqrt{-g}\left\{\frac{1}{\kappa^{2}}\left(R-\gamma R^{-n}+\zeta R^{2}\right)+\left(\frac{R}{\mu^{2}}\right)^{\alpha} L_{d}+L_{m}\right\} .
$$

Here $L_{d}$ describes dark or usual matter coupled with gravity and $\gamma$ and $\zeta$ are constants. The model admits the early time inflation and the late time dark energy universe in unified description like in phantom/quantum matter theory [16]. Let $L_{d}$ (which also may be interpreted as part of dark energy[15]) be the Lagrangian of the free massless scalar $\varphi$ :

$$
L_{d}=-\frac{1}{2} g^{\mu v} \partial_{\mu} \varphi \partial_{v} \varphi
$$

For FRW universe where $\phi$ only depends on $t(\phi=\phi(t))$ and $L_{m}=0$, the solution of scalar field equation is given by (with constant $q$ ) $\dot{\varphi}=q a^{-3} R^{-\alpha}$.

When the curvature is small, the third $R^{2}$-term in (8) becomes dominant. If we assume the first and the second terms could be neglected and $L_{m}=0$, the equation of the motion given by the variation over $g_{00}$ has the following solution:

$$
a=a_{0} t^{\frac{\alpha+2}{3}} \quad\left(H=\frac{\alpha+2}{3 t}\right), \quad a_{0}^{6} \equiv-\frac{\kappa^{2} q^{2}(2 \alpha-1)(\alpha-1)}{27 \mu^{2 \alpha}(\alpha+2)(\alpha+1)^{\alpha-1}\left(\frac{2}{3}(2 \alpha-1)\right)^{\alpha+2}} .
$$

The effective $w$ is given by $w=-1+2 /(\alpha+2)$. If $\alpha>1(\alpha<0)$, the universe is accelerating (decelerating). On the other hand, when the curvature is small, the second $R^{-n}$ term in (8) becomes dominant. The solution is found to be

$$
\begin{aligned}
& a=a_{0} t^{\frac{\alpha-n}{3}} \quad\left(H=\frac{\alpha+2}{3 t}\right) \\
& a_{0}^{6} \equiv \frac{\kappa^{2} q^{2}(2 \alpha-1)(\alpha-1)}{3 \mu^{2 \alpha}(\alpha-n)\left(3+11 n+7 n^{2}-(2+n) \alpha\right)(\alpha+1)^{\alpha-1}\left(\frac{2}{3}(2 \alpha-1)\right)^{\alpha+2}},
\end{aligned}
$$

and the effective $w$ is given by $w=-1+2 /(\alpha-n)$. Then if $\alpha-n>3$, we have $w<-\frac{1}{3}$ and the universe is accelerating. Hence, for some parameters choice the effective $w$ may cross $w=-1$ border: from greater than -1 to $w<-1$. This is an interesting feature of gravitational dark energy. When $w<-1$, there usually occurs finite-time singularity. In this model, due to $R^{2}$-term (8) it does not occur eventually.

One may consider the case that $L_{m} \neq 0$. We also assume the Hubble constant behaves as (which defines behaviour of matter from field equations) $H=h_{0}+h_{1}\left(t_{s}-t\right)^{m}$. The equation given by the variation over $g_{00}$ in (8) contains the terms including $\frac{d^{2} H}{d t^{2}}$ but not the terms including $\frac{d^{k} H}{d t^{k}}$ $(k \geq 3)$. Therefore if $m>2$, the matter energy density $\rho$ should be finite:

$$
\rho \rightarrow \rho_{0} \equiv \frac{6}{\kappa^{2}}\left\{h_{0}^{2}-(2+n)\left(12 h_{0}^{2}\right)^{-n-1}\right\}-\frac{q^{2}\left(12 h_{0}^{2}\right)^{-\alpha}(\alpha+2)}{4 \mu^{2 \alpha} a_{0}^{6}} .
$$

Here $a_{0}$ is the size of the universe at $t=t_{s}$. As $\rho>0$ usually, $\rho_{0}$ should be also positive. The equation given by the variation over $g_{i j}$ contains the terms including $\frac{d^{3} H}{d t^{3}}$. Therefore if $3>m>2$, 
since $\frac{d^{3} H}{d t^{3}}$ diverges, the matter pressure $p$ diverges:

$p \rightarrow p_{0} \equiv 6 h_{1}\left[-\frac{1}{\kappa^{2}}\left\{n(n+1) \gamma\left(12 h_{0}\right)^{-n-2}+2 \zeta\right\}-\frac{\zeta \alpha(\alpha-1) q^{2}}{2 \mu^{\alpha} a_{0}^{6}\left(12 h_{0}^{2}\right)^{\alpha-2}}\right] m(m-1)(m-2)\left(t_{s}-t\right)^{m-3}$.

In case of the Einstein gravity with matter, such a sudden singularity appears when $0<m<1$ [17] (see also [18]). The theory under consideration moderates the singularity.

Let us finally consider the role of $L_{d}$ in (8) when it describes the scalar field $\varphi$ with the potential $V(\varphi)$. For simplicity, we put $\gamma=L_{m}=0$. When the scalar field $\varphi$ only depends on time, one has

$$
\rho_{d}=\frac{1}{2} \dot{\varphi}^{2}+V(\varphi), \quad L_{d}=p_{d}=\frac{1}{2} \dot{\varphi}^{2}-V(\varphi) .
$$

Varying over $g_{00}$ gives:

$$
\begin{aligned}
0= & -\frac{3 H^{2}}{\kappa^{2}}+\frac{1}{\mu^{2 \alpha}}\left[3 \alpha\left(6 \frac{d H}{d t}+12 H^{2}\right)^{\alpha-1}\left(\frac{d H}{d t}+H^{2}\right) p_{d}\right. \\
& \left.-3 \alpha H \frac{d}{d t}\left\{\left(6 \frac{d H}{d t}+12 H^{2}\right)^{\alpha-1} p_{d}\right\}+\frac{1}{2}\left(6 \frac{d H}{d t}+12 H^{2}\right)^{\alpha} \rho_{d}\right] .
\end{aligned}
$$

The variation over $g_{i j}$ looks like

$$
\begin{aligned}
0= & \frac{1}{\kappa^{2}}\left(2 \frac{d H}{d t}+3 H^{2}\right)+\frac{1}{\mu^{2 \alpha}}\left[\left\{(3-\alpha) \frac{d H}{d t}+(6-3 \alpha) H^{2}\right\}\left(6 \frac{d H}{d t}+12 H^{2}\right)^{\alpha-1} p_{d}\right. \\
& \left.+\left(\frac{d^{2}}{d t^{2}}+2 H \frac{d}{d t}\right)\left\{\left(6 \frac{d H}{d t}+12 H^{2}\right)^{\alpha-1} p_{d}\right\}\right] .
\end{aligned}
$$

We now assume $H=h_{0} / t$ (when $h_{0}>0$ ) or $H=-h_{0} /\left(t_{s}-t\right)$ (when $h_{0}<0$ ). Then the solution of (15) and (16) is found to be

$$
\begin{aligned}
p_{d} & =p_{0} t^{2 \alpha-2}, \quad \rho_{d}=\rho_{0} t^{2 \alpha-2} \quad\left(h_{0}>0\right), \\
p_{d} & =p_{0}\left(t_{s}-t\right)^{2 \alpha-2}, \quad \rho_{d}=\rho_{0}\left(t_{s}-t\right)^{2 \alpha-2} \quad\left(h_{0}<0\right), \\
p_{0} & \equiv \frac{\mu^{2 \alpha}\left(2+3 h_{0}\right)}{\kappa^{2}\left(-6 h_{0}+12 h_{0}^{2}\right)^{\alpha-1}\left\{-3+\alpha+(6-3 \alpha) h_{0}\right\}}, \\
\rho_{0} & \equiv \frac{6 \mu^{2 \alpha} h_{0}\left\{2 \alpha+(3-6 \alpha) h_{0}-6(1-\alpha) h_{0}^{2}\right\}}{\kappa^{2}\left(-6 h_{0}+12 h_{0}^{2}\right)^{\alpha}\left\{-3+\alpha+(6-3 \alpha) h_{0}\right\}} .
\end{aligned}
$$

One may write

$$
\dot{\varphi}^{2}=\rho_{d}+p_{d}=\frac{\varphi_{0}^{2}}{\alpha^{2}} t^{2 \alpha-2} \text { or } \frac{\varphi_{0}^{2}}{\alpha^{2}}\left(t_{s}-t\right)^{2 \alpha-2}, \quad V(\varphi)=\frac{\rho_{d}-p_{d}}{2}=V_{0} t^{2 \alpha-2} \text { or } V_{0}\left(t_{s}-t\right)^{2 \alpha-2} .
$$

Then we find $\varphi=\varphi_{0} t^{\alpha}, V(\varphi)=V_{0}\left(\varphi / \varphi_{0}\right)^{2-\frac{2}{\alpha}}$. When $h_{0}<0$, if $\alpha \geq 1, \rho_{d}$ and $p_{d}$ does not diverge at $t=t_{s}$, which is different from the usual phantom matter generating the Big Rip singularity.

Thus, modified gravity which gives very natural explanation of dark energy (via expansion) may unify the early time inflation and late time dark energy universe and may pass solar system tests. The phantom phase (if any) may be transient here and future finite-time singularity may be avoided or (depending from matter/dark energy choice) it changes the structure. 


\section{References}

[1] S.M. Carroll, V. Duvvuri, M. Trodden and M.S. Turner, Is Cosmic Speed-Up Due to New Gravitational Physics?, Phys.Rev. D70 (2004) 043528, [astro-ph/0306438]; S. Capozzielo, S. Carloni and A. Troisi, Quintessence without scalar fields, astro-ph/0303041.

[2] S. Nojiri and S.D. Odintsov, Where new gravitational physics comes from: M-theory?, Phys.Lett. B576 (2003) 5, [hep-th/0307071].

[3] U. Guenther, A. Zhuk, V. Bezerra and C. Romero, AdS and stabilized extra dimensions in multidimensional gravitational models with nonlinear scalar curvature terms $1 / R$ and $R^{4}$, hep-th/0409112.

[4] T. Chiba, 1/R gravity and Scalar-Tensor Gravity, Phys.Lett. B575 (2003) 1, [astro-ph/0307338]; A.D. Dolgov and M. Kawasaki, Can modified gravity explain accelerated cosmic expansion?, Phys.Lett. bfB573 (2003) 1, [astro-ph/0307285]; M.E. Soussa and R.P. Woodard, The Force of Gravity from a Lagrangian containing Inverse Powers of the Ricci Scalar, Gen.Rel.Grav. 36 (2004) 855, astro-ph/0308114;

[5] S. Nojiri and S.D. Odintsov, Modified gravity with negative and positive powers of the curvature: unification of the inflation and of the cosmic acceleration, Phys.Rev. D68 (2003)123512, [hep-th/0307288].

[6] S. Nojiri and S.D. Odintsov, Modified gravity with $\ln R$ terms and cosmic acceleration, Gen.Rel.Grav. 36 (2004) 1765, [hep-th/0308176]; The minimal curvature of the universe in modified gravity and conformal anomaly resolution of the instabilities, Mod.Phys.Lett. A19 (2004) 627, hep-th/0310045.

[7] P. Wang and X. Meng, Palatini Formulation of Modified Gravity with $\ln R$ Terms, Phys.Lett. B584 (2004)1, [hep-th/0309062]; Palatini formulation of modified gravity with squared scalar curvature, astro-ph/0308284; Palatini formulation of the $R^{-1}$ modified gravity with an additionally squared scalar curvature term, gr-qc/0411007.

[8] P. Wang and X. Meng, Palatini formulation of $L(R)$ gravity, astro-ph/0406455; D. Vollick, On the viability of the Palatini form of 1/R gravity, Class.Quant.Grav. 21 (2004) 3813, [gr-qc/0312041]; E. Flanagan, The conformal frame freedom in theories of gravitation, Class.Quant.Grav. 21 (2004) 3817, [gr-qc/0403063]; G. Olmo and W. Komp, Nonlinear Gravity Theories in the Metric and Palatini Formalisms, gr-qc/0403092; G. Allemandi, A. Borowiec and M. Francaviglia, Accelerated Cosmological Models in First-Order Non-Linear Gravity, Phys.Rev. D70 (2004) 043524, [hep-th/0403264]; Y.Ezawa, H.Iwasaki, Y.Ohkuwa, T.Uegaki, N.Yamada, T.Yano, Interpretation of the first order formalism of $f(R)$-type gravity and the corresponding second order formalism, gr-qc/0405054; A. Dominguez and D. Barraco, Newtonian limit of the singular $f(R)$ gravity in the Palatini formalism, Phys.Rev. D70 (2004) 043505, [gr-qc/0408069]; S. Capozziello, V.F. Cardone and M. Francaviglia, $f(R)$ theories of gravity in Palatini approach matched with observations, astro-ph/0410135; P. Wang, G. Kremer, D. Alves and X. Meng, A Note on Energy-Momentum Conservation in Palatini Formulation of L(R) Gravity, gr-qc/0408058.

[9] G. Allemandi, A. Borowiec and M. Francaviglia, Accelerated Cosmological Models in Ricci squared Gravity, Phys.Rev. D70 (2004) 103503, [hep-th/0407090]; G. Allemandi, M. Capone, S. Capozziello and M. Francavilia, Conformal aspects of Palatini approach in Extended Theories of Gravity, hep-th/0409198; S. Carroll, A. De Felice, V. Duvvuri, D. Easson, M. Trodden and M. Turner, The Cosmology of Generalized Modified Gravity Models, astro-ph/0410031; S. Carloni, P.K. Dunsby, S. Capozziello and A. Troisi, Cosmological dynamics of $R^{n}$ gravity, gr-qc/0410046. 
[10] N. Furey and A. DeBenedictis, Wormhole throats in $R^{m}$ gravity, gr-qc/0410088; I. Brevik, S. Nojiri, S.D. Odintsov and L. Vanzo, Entropy and universality of Cardy-Verlinde formula in dark energy universe, Phys.Rev. D70 (2004) 043520, [hep-th/0401073].

[11] M.C.B. Abdalla, S. Nojiri, S.D. Odintsov, Consistent modified gravity: dark energy, acceleration and the absence of cosmic doomsday, hep-th/0409177.

[12] G. Cognola and S. Zerbini, Minisuperspace Approach of Generalized Gravitational Models, gr-qc/0407103; V. Faraoni, gr-qc/0407021; A. Nunez and S. Solganik, The content of $f(R)$ gravity, hep-th/0403159.

[13] J.D. Barrow and S. Cotsakis, Inflation and the conformal structure of higher-order gravity theories, Phys.Lett. B214 (1988) 515.

[14] R. Caldwell, M. Kamionkowski and N. Weinberg, Phantom Energy and Cosmic Doomsday, Phys.Rev.Lett. 91 (2003) 071301, [astro-ph/0302506]; B. McInnes, The dS/CFT Correspondence and the Big Smash, JHEP 0208 (2002) 029, [hep-th/0112066]; P. Gonzalez-Diaz, K-Essential Phantom Energy: Doomsday around the corner?, Phys.Lett. $\mathbf{B 5 8 6}$ (2004) 1, [astro-ph/0312579]; M. Sami and A. Toporensky, Phantom Field and the Fate of Universe, Mod.Phys.Lett. A19 (2004) 1509, [gr-qc/0312009]; P. Gonzales-Diaz and C. Siguenza, Phantom thermodynamics, Nucl.Phys. B697 (2004) 363, [astro-ph/0407421]; L.P. Chimento and R. Lazkoz, On big rip singularities, Mod.Phys.Lett. A19 (2004) 2479, [gr-qc/0405020]; E. Elizalde, S. Nojiri and S.D. Odintsov, Late-time cosmology in (phantom) scalar-tensor theory: dark energy and the cosmic speed-up, Phys.Rev. D70 (2004) 043539, [hep-th/0405034]; S. Nojiri and S.D. Odintsov, The final state and thermodynamics of dark energy universe, hep-th/0408170; G. Calcagni, Patch dualities and remarks on nonstandard cosmologies, gr-qc/0410027; P. Wu and H. Yu, Active Galaxies and Cluster Gas, astro-ph/0407484; S. Nesseris and L. Perivolaropoulos, The Fate of Bound Systems in Phantom and Quintessence Cosmologies, astro-ph/0410309; P. Scherrer, Phantom Dark Energy, Cosmic Doomsday, and the Coincidence Problem, astro-ph/0410508; Z. Guo,Y. Piao, X. Zhang and Y. Zhang, Cosmological Evolution of a Quintom Model of Dark Energy, astro-ph/0410654; R.G. Cai and A. Wang, Cosmology with Interaction between Phantom Dark Energy and Dark Matter and the Coincidence Problem, hep-th/0411025; Y. Wei, Late-time phantom universe in $\mathbf{S O}(\mathbf{1}, \mathbf{1})$ dark energy model with exponential potential, gr-qc/0410050; M. Dabrowski and T. Stachowiak, Generalized phantom cosmologies, hep-th/0411199; H. Stefancic, "Expansion" around the vacuum equation of state - sudden future singularities and asymptotic behavior, astro-ph/0411630.

[15] S. Nojiri and S.D. Odintsov, Gravity assisted dark energy dominance and cosmic acceleration, Phys.Lett. B599 (2004) 137, astro-ph/0403622.

[16] S. Nojiri and S.D. Odintsov, Quantum deSitter cosmology and phantom matter, Phys.Lett. B562 (2003) 147, [hep-th/0303117].

[17] J.D. Barrow, Sudden Future Singularities, Class.Quant.Grav. 21 (2004) L79, [gr-qc/0403084].

[18] J.D. Barrow, More General Sudden Singularities, Class.Quant.Grav. 21 (2004) 5619, [gr-qc/0409062]; S. Cotsakis and I. Klaoudatou, Future Singularities of Isotropic Cosmologies, gr-qc/0409022; V. Sahni and Yu. Shtanov, Braneworld models of dark energy, JCAP 0311 (2003) 014, [astro-ph/0202346]; S. Nojiri and S.D. Odintsov, Quantum escape of sudden future singularity, Phys.Lett. B595 (2004) 1, [hep-th/0405078]; K. Lake, Sudden future singularities in FLRW cosmologies, Class.Quant.Grav. 21 (2004) L129, [gr-qc/0407107]; M. Dabrowski, Inhomogenized sudden future singularities, gr-qc/0410033; L. Fernandez-Lambrina and R. Lazkoz, Geodesic behaviour of sudden future singularities, gr-qc/0410124; J.D. Barrow and C. Tsagas, New Isotropic and Anisotropic Sudden Singularities, gr-qc/0411045. 